\title{
El uso pedagógico de las tecnologías de la información y la comunicación (TIC) y su influencia en el aprendizaje significativo de estudiantes de media técnica en Zipaquirá, Colombia*
}

\author{
Wilder Banoy Suarez ${ }^{\mathrm{a}}$
}

\begin{abstract}
Resumen: Este artículo presenta los resultados de una investigación doctoral desarrollada entre el 2014 y el 2017. Tuvo como objetivo determinar en qué medida el uso pedagógico de las tecnologías de la información y la comunicación (TIC) influía en el aprendizaje significativo de estudiantes de Zipaquirá, Colombia, en el marco del proyecto pedagógico "Empresarios en la Nube, Community Managers en la media técnica". Se realizó una profunda revisión de estado del arte y un marco teórico, tanto de la variable independiente como de la variable dependiente. Esta revisión involucró un número final de 258 fuentes oficiales en el documento. En términos metodológicos, el diseño de la investigación es experimental, en un nivel cuasiexperimental, con un enfoque mixto. Se especifica, además, la fase de análisis de resultados, de acuerdo con las técnicas de recolección, con el fin de sustentar los pormenores de medición de indicadores y las dimensiones de cada variable. Los resultados revelaron un vínculo directo entre el uso pedagógico de las Tic y el mejoramiento del nivel de aprendizaje significativo en el grupo experimental de la muestra; la síntesis general de análisis, la discusión y las conclusiones presentadas así lo concretan.
\end{abstract}

Palabras clave: educación; enseñanza; TIC; aprendizaje significativo; motivación; flipped learning.

Recibido: 18 marzo 2019 - Evaluado: 13 junio 2019 - Aceptado: 15 junio 2019

* Artículo de investigación, producto de una tesis doctoral de quinientas treinta y tres páginas, finalizada y sustentada presencialmente en México en julio del 2017, titulada "El uso pedagógico de las tic y su influencia en el aprendizaje significativo de los estudiantes de Zipaquirá, Colombia, en el año 2015". La investigación se desarrolló entre el 2014 y el 2017, y se realizó para obtener el título de Doctor en Educación con la Universidad de Baja California, México.

a Doctor en Educación, Magíster en Gestión de Organizaciones, Especialista en Alta Gerencia, Especialista en Informática y Telemática, Licenciado en Diseño Tecnológico. Docente del proyecto pedagógico "Empresarios en la Nube, Community Managers en la media técnica" — base de la investigación — con la Secretaría de Educación de Zipaquirá, Colombia. Docente investigador y líder del semillero de investigación Clase Invertida, innovación educativa, en la Universidad Minuto de Dios, Colombia.

E-mail: wilder.banoy@uniminuto.edu.co

b Esta investigación y el diseño pedagógico inherente a ella le ha permitido al autor representar a Colombia en Incheon, Seúl (Corea del Sur), en el congreso mundial de educadores expertos de Microsoft Seattle USA-2015, ser ponente por Colombia en el congreso internacional Virtual Educa, Colombia 2017; obtener el primer puesto de Colombia como experiencia e investigación Innovadora en el Evento Educa Digital 2017 y la condecoración otorgada por el presidente de la República en la noche de excelencia docente en diciembre del 2017. 


\title{
Pedagogical Use of Information and Communication Technologies (ICTS) and Their Influence on the Significant Learning of Technical High School Students in Zipaquirá, Colombia
}

\begin{abstract}
This article presents the results of doctoral research carried out between 2014 and 2017. Its aim is to determine to what extent the pedagogical use of information and communication technologies (ICTs) influenced the significant learning of students in Zipaquirá, Colombia, within the framework of the pedagogical project "Cloud Entrepreneurs, Community Managers in Technical High School Education." A thorough literature review and a theoretical framework of both the independent and dependent variables were prepared. This review involved a final number of 258 official sources in the document. As to the method, the research design is experimental, on a quasi-experimental level, with a mixed approach. The result analysis phase is also specified, according to data collection techniques, in order to back up the details of indicator measurement and the dimensions of each variable. Results revealed a direct link between the pedagogical use of ICTs and the improvement of significant learning in the experimental group of the sample; the summarized analysis, discussion, and conclusions presented so prove it.
\end{abstract}

Keywords: Education; teaching; ICTs; significant learning; motivation; flipped learning.

O uso pedagógico das tecnologias da informação e da comunicação (TIC) e sua influência na aprendizagem significativa de estudantes de média técnica em Zipaquirá, Colômbia

Resumo: Este artigo apresenta resultados de uma pesquisa de doutorado desenvolvida entre 2014 e 2017. O objetivo foi determinar em que medida o uso pedagógico das tecnologias da informação e da comunicação (TIC) influenciava na aprendizagem significativa de estudantes de Zipaquirá, Colômbia, no âmbito do projeto pedagógico "Empresários na nuvem, Community Managers na média técnica". Foram realizados uma revisão exaustiva do estado da arte e um referencial teórico, tanto da variável independente quanto da dependente. Essa revisão envolveu 258 fontes oficiais no documento. Em termos metodológicos, o desenho da pesquisa é experimental, em um nível quase -experimental, com abordagem mista. Além disso, é especificada a fase de análise de resultados de acordo com as técnicas de coleta, a fim de apoiar a medição de indicadores e as dimensões de cada variável. A síntese geral da análise, a discussão e as conclusões apresentadas demonstraram um vínculo direto entre o uso pedagógico das TIC e a melhoria do nível de aprendizagem significativa no grupo experimental da amostra.

Palavras-chave: educação; ensino; TIC; aprendizagem significativa; motivação; flipped learning. 


\section{Introducción}

Un gran desafío que tiene el mundo actual es innovar en educación (Robinson, 2006); el reto se hace evidente en modelos educativos tradicionales permeados por falta de interés y motivación al aprender. En este sentido, Robinson y Aronica afirman: "Desde que nacen, los niños tienen una sed de aprendizaje inagotable. Para muchos de ellos, demasiados, su paso por la escuela va apagando esa sed" (2016, p. 18). De ahí que ese tipo de escuela deba adaptarse a los cambios acelerados propios del mundo actual, ya que "son muy pocas las áreas que quedan fuera del alcance de los medios digitales, cuya influencia futura promete ser de similar envergadura e igualmente difícil de prever" (Gardner y Davis, 2014, p. 19).

La razón, según Cabero y Aguaded (2013), se sustenta en que "han cambiado tanto las tecnologías como las características de los destinatarios de la instrucción, y los contextos de formación" (p. 15). Esa es la razón de ser de este artículo, que sintetiza el proceso investigativo desarrollado por el autor en Zipaquirá, Colombia, y su pertinencia con instituciones educativas de características similares.

\section{Contexto de la investigación y situación problémica}

Se investigó sobre la posibilidad de mejorar y optimizar durante el 2015 resultados de estrategias de enseñanza y aprendizaje tradicionales o magistrocentristas, en las cuales se privilegiaba el papel del maestro al considerarlo el eje central de todo el proceso (Zubiría, 2006, p. 15). Para la comunidad investigada no era común que se empleara la tecnología como un medio con el fin de motivar y mejorar los niveles de atención de los estudiantes frente a contenidos específicos y contextualizados en su realidad. Tampoco era común que se empleara para generar engagement educativo o "implicación escolar, compromiso y enganche" (Zárrate, 2016), y que este derivara en un aprendizaje significativo; entendido como una teoría psicológica que "pone el énfasis en lo que ocurre en el aula cuando los estudiantes aprenden; en la naturaleza de ese aprendizaje; en las condiciones que se requieren para que éste se produzca; en sus resultados y, consecuentemente, en su evaluación" (Ausubel, 1976).

En este orden de ideas, el contexto del experimento se enmarcó en características particulares de la institución educativa -fase diagnósticaque dieron origen a la investigación. Entre otras, la falta de motivación de los estudiantes por la clase magistral tradicional, el aprendizaje sin trascendencia y significado, hábitos de estudio ausentes, uso deficiente de dispositivos y servicios tecnológicos, falta de formación docente en metodologías disruptivas de enseñanza, avance nulo en el uso de recursos destinados a innovación educativa, bajo poder adquisitivo de servicios y productos de orden tecnológico por parte de las familias y falta de inversión gubernamental en infraestructura, formación e investigación.

Ahora bien, el problema de investigación general y los problemas específicos se ligan a objetivos e hipótesis, con respeto, por supuesto, por la coherencia del estudio y su relación con los resultados. Dicho de otro modo, el problema está "formulado claramente y sin ambigüedad mediante una pregunta o varias que orientan hacia las respuestas que se buscan en la investigación" (Kerlinger y Lee, citados por Sabariego y Bisquerra, 2004, p. 95).

El problema es de investigación o de conocimiento, ya que busca exponer la influencia de la variable independiente sobre la dependiente. En segunda instancia, es práctico, por ser experimental en educación. De forma concreta y precisa, la formulación del problema es: ¿en qué medida el uso pedagógico de las TIC influye en el aprendizaje significativo de los estudiantes de la media técnica de Zipaquirá, Colombia, si se tienen en cuenta las condiciones reales del contexto y se aplica una investigación de nivel cuasiexperimental?

\section{Objetivos}

Comprendidos como "los mandatos que se da el investigador, con relación al objeto de su investigación [...], derivan del problema identificado" (Gómez, Deslauriers y Alzate, 2010, p. 57). El 
objetivo general se sintetiza en determinar en qué medida el uso pedagógico de las TIC influye en el aprendizaje significativo de los estudiantes de la media técnica de Zipaquirá, Colombia, si se tienen en cuenta las condiciones reales del contexto y se aplica una investigación de nivel cuasiexperimental.

\section{Objetivos específicos}

Los objetivos específicos se vinculan de forma directa a las conclusiones y son:

- Establecer la influencia del uso pedagógico de las TIC sobre la motivación de los estudiantes en las dinámicas propias de clase tanto en el aula como fuera de ella.

- Determinar si el uso pedagógico de las Tic propende a la identificación y solución de problemas cotidianos de los estudiantes vinculados con los contenidos y las prácticas académicas propias de la clase.

- Identificar la forma en que el uso pedagógico de las TIC modifica los hábitos de aprendizaje, lectura y pensamiento crítico de los estudiantes.

- Determinar cómo el uso pedagógico de las TIC posibilita un aprendizaje con significado para los estudiantes.

- Determinar en qué medida el uso pedagógico de las TIC influye en las actividades tradicionales desarrolladas por los estudiantes en su tiempo libre.

\section{Marco teórico y estado del arte}

El producto académico se fundamentó en trece antecedentes teóricos, doce antecedentes de campo y el análisis de conceptos que se vincularon con el eje central de la investigación y sus variables fundamentales. En ese orden, se estudiaron minuciosamente conceptos ligados a cada dimensión tanto de la variable independiente (vI) como de la variable dependiente (vD); el resultado fue un marco de referencia de 215 páginas de las 533 del documento final.

\section{Uso pedagógico de las TIC (variable independiente)}

Tabla 1. Operacionalización de variable independiente

\begin{tabular}{|c|c|c|}
\hline V. independiente & Dimensiones & Indicadores \\
\hline \multirow{13}{*}{$\begin{array}{l}\text { Uso pedagógico } \\
\text { de las TIC }\end{array}$} & \multirow{3}{*}{$\begin{array}{l}\text { Aprendizaje virtual y comunicación } \\
\text { online }\end{array}$} & Percepción de la relación entre el uso de las TIC y el aprendizaje \\
\hline & & Desarrollo de tareas vinculadas al aprendizaje virtual \\
\hline & & Relación entre internet y aprendizaje \\
\hline & \multirow{4}{*}{$\begin{array}{l}\text { TIC en el aula y nuevas metodologías de } \\
\text { enseñanza aprendizaje }\end{array}$} & Nivel de uso e implementación de las TIC en el aula \\
\hline & & Posibilidad de cambio de metodología de enseñanza y aprendizaje \\
\hline & & Evolución de estrategias de enseñanza y aprendizaje \\
\hline & & Cambios en la metodología de enseñanza y aprendizaje \\
\hline & \multirow{2}{*}{$\begin{array}{l}\text { Competencias en el uso de las TIC } \\
\text { (estudiantes y docentes) }\end{array}$} & $\begin{array}{l}\text { Conocimiento de procesos básicos que involucran las tic en } \\
\text { situaciones cotidianas }\end{array}$ \\
\hline & & Actualización y capacitación en procesos de las TIC \\
\hline & \multirow{2}{*}{ Redes sociales y educación } & Uso de redes sociales \\
\hline & & Empleo de redes sociales virtuales en educación \\
\hline & \multirow{2}{*}{ Acceso a las TIC y su uso cotidiano } & Acceso a internet, equipos informáticos y redes sociales \\
\hline & & Uso cotidiano de tic en labores académicas \\
\hline
\end{tabular}

Fuente: elaboración propia. 


\section{Aprendizaje significativo (variable dependiente)}

Tabla 2. Operacionalización de variable dependiente

\begin{tabular}{|c|c|c|}
\hline Variable dependiente & Dimensiones & Indicadores \\
\hline \multirow{13}{*}{$\begin{array}{l}\text { Aprendizaje } \\
\text { significativo }\end{array}$} & \multirow{3}{*}{ Motivación (engagement) } & Percepción de clase tradicional \\
\hline & & Relación entre el uso pedagógico de las TIC y motivación \\
\hline & & $\begin{array}{l}\text { Relación entre el uso de TIC y motivación de los estudiantes en procesos } \\
\text { académicos }\end{array}$ \\
\hline & \multirow{3}{*}{$\begin{array}{l}\text { Resolución de problemas y } \\
\text { trabajo por proyectos }\end{array}$} & Identificación y solución de problemas reales \\
\hline & & $\begin{array}{l}\text { Resolución de problemas cotidianos empleando las tic en procesos } \\
\text { académicos }\end{array}$ \\
\hline & & Relación entre la realidad del estudiante y lo aprendido en el aula \\
\hline & $\begin{array}{l}\text { Conocimientos previos y } \\
\text { nuevo aprendizaje }\end{array}$ & Aprendizaje real frente al paso del tiempo \\
\hline & \multirow{3}{*}{$\begin{array}{l}\text { Hábitos de aprendizaje, } \\
\text { lectura y pensamiento } \\
\text { crítico }\end{array}$} & Uso tradicional de las TIC en procesos de aprendizaje autónomo \\
\hline & & Comprensión de lectura \\
\hline & & Preferencias para acceder a la información \\
\hline & Estilos de aprendizaje & Reconocimiento de estilos de aprendizaje \\
\hline & \multirow{2}{*}{ Tiempo libre y uso de las TIC } & Actividades tradicionales en tiempo libre \\
\hline & & Uso de tecnología en tiempo de ocio \\
\hline
\end{tabular}

Fuente: elaboración propia.

\section{Metodología}

Vale la pena aclarar que "según sea el marco teórico en que se basa la investigación, será la propuesta metodológica para acercarse al objeto de estudio y construir el problema científico, los objetivos, las hipótesis, y para realizar los demás procesos de la investigación” (Rojas, 2013, p. 191.); lo anterior, permite inferir que la metodología engloba los respectivos métodos seleccionados por el investigador.

\section{Diseño, clasificación y enfoque de la investigación}

La investigación es de carácter experimental debido a que se actuó sobre los fenómenos a investigar (Cerda, 2008, p. 53). Asimismo, de orden cuasiexperimental y con enfoque mixto, en el que se mezclan o combinan técnicas de investigación, métodos, enfoques, conceptos o lenguaje cuantitativo o cualitativo en un solo estudio (Johnson y Onwuegbuzie, citados por Pereira, 2011, p. 18). Se manipuló la variable independiente en el hábitat de los estudiantes, con el fin de analizar su efecto sobre la variable dependiente. Lo anterior, al ejercer el análisis comparativo de los resultados obtenidos entre los grupos de la muestra.

Es pertinente mencionar que se tuvo en cuenta el principio de "presencia o ausencia". Este nivel o grado implica que "un grupo se expone a la presencia de la variable independiente y el otro no. Posteriormente, los dos grupos se comparan para identificar disimilitudes con el grupo que no fue expuesto" (Hernández, Fernández y Baptista, 2014). Todo con un tratamiento de preprueba y posprueba en los dos grupos.

Es un estudio prospectivo debido a que, como lo afirman Pineda, De Alvarado y De Canales, la información se registró a medida que ocurrieron los fenómenos propios de la aplicación de la metodología en el grupo experimental de la muestra y su influencia sobre el aprendizaje significativo de los estudiantes (1994, p. 83). No se indagó sobre hechos ocurridos en el pasado; es una investigación 
de campo y, de acuerdo con el lugar de ejecución, los estudios se realizaron de forma vivencial en el hábitat de la muestra.

Se implementó un diseño preprueba, posprueba y de control; el eje principal se ajustó a pruebas dirigidas a los estudiantes, mientras que los otros instrumentos sustentaron el contexto de los sujetos en todo el proceso, y tuvieron en cuenta a docentes y padres de familia. El enfoque de la investigación fue mixto, de ahí que las técnicas de recolección empleadas fueran cuantitativas: pruebas (test), encuestas (cuestionarios), y cualitativas: observación participante general (holística), grupos de enfoque (entrevistas semiestructuradas) y prueba final comparativa de aprendizaje para la muestra. Ambos enfoques se complementaron y, tal como lo describen Hernández et al. (2014), la meta era "utilizar fortalezas de ambos tipos de indagación, combinándolos y tratando de minimizar sus debilidades potenciales".

\section{Población y muestra}

El universo de estudio o la población apuntó a 245 estudiantes de la media técnica en Gestión Empresarial de la Institución Educativa Municipal Cundinamarca de Zipaquirá, Colombia (entidad pública) en el 2015. El tipo de muestreo fue no probabilístico o por conveniencia, ya que eran grupos puros, es decir, no se modificaron sus características, no se asignaron al azar ni fueron emparejados, todo se fundamentó en la conveniente accesibilidad y proximidad de los sujetos para el investigador (Otzen y Manterola, 2017, p. 230); en concreto, fueron 80 estudiantes, distribuidos en dos grupos: control y experimental. Cabe resaltar que se eligieron estudiantes de este nivel en razón a que no habían compartido escenario académico alguno con el investigador, lo cual se realizó con el fin de mejorar el nivel de validez interna.

Las asignaturas en las que se desarrolló el experimento y el trabajo de campo fueron Tecnología e Informática y Diseño Publicitario Empresarial; estas se fusionaron, desde el 2014, con el propósito de formar el proyecto pedagógico "Empresarios en la Nube, Community Managers en la Media Técnica", formulado por el autor para la investigación (Banoy, 2015, p. 11).
El grupo de control presenció procesos pedagógicos basados en clase magistral y material pedagógico tradicional o convencional, mientras que el uso pedagógico de las Tic se aplicó en el grupo experimental como medio para abordar contenidos, prácticas y evaluaciones. Es pertinente aclarar que el proyecto se cimentó, entre otros, gracias a los elementos y las herramientas que se decriben a continuación.

Un canal de YouTube propio con videos vinculados a las temáticas a tratar, material diseñado y elaborado por el autor para la metodología clase invertida o flipped learning (Banoy, 2013), la cual se concibe como "un enfoque pedagógico en el que la instrucción directa se desplaza de la dimensión del aprendizaje grupal a la dimensión del aprendizaje individual" (FNL, 2014, p. 1). Para el trabajo telecolaborativo con el grupo experimental - comunicación, realimentación y socializaciónse emplearon distintas redes sociales: Facebook, como aula virtual, perfil, dos páginas (fan pages) para asignaturas y un grupo cerrado, Pinterest, con tableros destinados al proyecto pedagógico, $y$ Twitter.

Asimismo, fuentes externas para aprendizaje online - grupo experimental- como soporte a los contenidos tratados. En este propósito se utilizaron plataformas de cursos masivos abiertos y en línea denominados "MOoc" por sus siglas en inglés (massive open online courses) (Bartolomé y Steffens, 2015, p. 92) y otros. En resumen, el uso pedagógico de las TIC se fundamentó en el empleo de una gran cantidad de herramientas utilizadas como medio para acceder a los contenidos, desarrollar prácticas e intentar potenciar el aprendizaje significativo, principalmente, con la metodología de clase invertida o flipped learning. Fueron más de 40 herramientas virtuales que sirvieron como medio al propósito de la investigación.

\section{Técnicas e instrumentos}

\section{Técnica cuantitativa 1. Test de estilos de aprendizaje}

El test de estilos de aprendizaje se basó en el modelo propuesto por Richard M. Felder y Linda 
K. Silverman. Este instrumento "evalúa preferencias de aprendizaje, dependiendo del perfil cognitivo del sujeto al que se le aplica" (Del V. Ramírez y Rosas, 2014, p. 180). Suministró indicadores que permitieron investigar, reforzar, reelaborar, alternar o cambiar las metodologías de enseñanza en las clases. Se aplicó en coordinación con el Centro de Investigación de la Universidad Nacional de Colombia, el Centro de Innovación Educativa Regional del Ministerio de Educación Nacional y el investigador.

En la metodología utlizada con respecto a estilos de aprendizaje de la muestra se tuvieron en cuenta cuatro dimensiones: "Sensitiva/intuitiva", "Visual/verbal", "Secuencial/global" y "Activa/ reflexiva”. Un total de 44 preguntas respondidas según preferencias del investigado, expresadas en conceptos de equilibrio apropiado, preferencia moderada (más) y preferencias muy fuertes (mucho más). Este último grupo requiere de gran atención así sea bajo el porcentaje. El test se aplicó por medio de formularios de Google a la totalidad de la media técnica del 2014; su objetivo era caracterizar la población para el 2015.

\section{Técnica cuantitativa 2.}

\section{Encuestas (cuestionarios)}

Se aplicaron a los dos grupos de la muestra en dos momentos: antes y después de la modificación de la variable independiente en el grupo de experimental. Es un diseño de preprueba, posprueba y grupo de control, $y$, puntualmente:

este diseño incorpora la administración de prepruebas a los grupos que componen el experimento [...]. La adición de la prueba previa ofrece dos ventajas, sus puntuaciones sirven para fines de control del experimento [...]. El diseño elimina el impacto de todas las fuentes de invalidación interna y la administración de pruebas queda controlada, ya que si la prueba afecta las puntuaciones de la posprueba lo hará de manera similar en ambos grupos (Hernández et al., 2014)

Cabe aclarar que los estudiantes, los docentes, los directivos y los padres de familia diligenciaron este tipo de instrumento en distintas temáticas y momentos, para lo cual tuvieron en cuenta, claro está, las dos variables que sustentan la estructura de la investigación. La primera encuesta se dirigió al total de padres de familia de estudiantes de la media técnica. Su objetivo fue identificar las características propias del contexto cercano de los estudiantes desde la perspectiva parental, e identificar la relación con la variable dependiente de la investigación.

Esta encuesta la diseñó, en parte, el grupo de investigación del CIER zona centro y la Facultad de Ingeniería de la Universidad Nacional de Colombia; las preguntas de la tercera sección las diseñó el investigador a fin de vincularlas directamente con las variables, las dimensiones y los indicadores. El proceso lo aplicó el autor en el último trimestre del 2014.

La segunda encuesta, dirigida a la muestra, tuvo como objetivo reconocer la percepción, la posición y la situación de los estudiantes frente a las dimensiones que hacen parte de las variables a investigar, de acuerdo con el tipo de aprendizaje, el modelo pedagógico presente en las distintas áreas y el uso de tecnología en el aula y fuera de ella, principalmente, en las dos asignaturas que hacen parte de la investigación.

La tercera encuesta, denominada "Uso de tecnología en el aula por parte de docentes de la media técnica", tuvo como objetivo identificar el nivel de uso de TIC en el aula por parte de docentes y generar valoraciones que sirvieran de soporte a la triangulación, comprendida como "el uso de varios métodos - tanto cualitativos como cuantitativosde fuentes de datos, de teorías, de investigadores, o de ambientes en el estudio de un fenómeno" (Okuda y Gómez, 2005, p. 119), con respecto a la encuesta principal dirigida a estudiantes.

\section{Técnica cualitativa 1. Observación participante general (holística)}

Instrumento sometido a dos pruebas piloto en junio del 2014, con dos grupos. Se modificó con el fin de brindar mayor claridad y orden en el registro de los datos, y así realizar una síntesis concreta de los fenómenos propios de la clase. Al finalizar la aplicación de 12 formatos durante el 2015, se realizó la prueba de aprendizaje de salida con 50 ítems 
- dos grupos - desarrollada en octubre, y así estar en capacidad de contrastar resultados.

\section{Técnica cualitativa 2. Grupos de enfoque (focus group)}

Esta técnica también se considera un tipo de entrevista grupal (tres a diez personas). Su característica principal "es la interacción entre los participantes y el uso analítico potencial de esta interacción" (Wilkinson, citado por Cabiria, 2012, p. 130). Esta técnica, aplicada en dos momentos, aportó a la investigación la posibilidad de recolectar datos pertinentes o preliminares que permitieron sustentar posibles puntos de cuestionarios -instrumentos cuantitativos- y comprender causas y consecuencias de fenómenos particulares. Los grupos de enfoque para la investigación no sobrepasaron los diez participantes, a pesar de que, como afirman Krueger y Casey, el formato y la naturaleza de la sesión o de las sesiones puede variar: depende del objetivo, de las características, de los participantes y del planteamiento del problema (citados por Hernández et al., 2009, p. 409).

Las guías, de forma similar a la técnica de entrevista, fueron semiestructuradas, a fin de otorgar un cierto margen de libertad y abordar temas emergentes, sin abandonar el eje central de cada actividad, claro está. Las sesiones se titularon de la siguiente manera:

- percepción de modelos de clase por parte de los estudiantes;

- influencia de tecnología en el proceso de enseñanza aprendizaje;

- diagnóstico sobre hábitos de estudio y uso cotidiano de tecnología;

- uso de tecnología en el aula por parte de docentes (se diseñó, pero no se aplicó, al considerar como suficiente ilustración la información recolectada con el cuestionario), y

- diagnóstico sobre percepción de TIC en el aula por parte de los padres de familia.

\section{Validación de instrumentos}

La validación de encuestas e instrumentos se dio por dos pruebas piloto aplicadas en septiembre del 2014, con tres grupos de diez estudiantes de la población. Al culminar, se rediseñaron preguntas y se suprimieron las que no se vinculaban de forma directa con el análisis de las variables, de las dimensiones y de los indicadores. La validación de expertos se llevó a cabo con dos docentes investigadoras colombianas que conocían el contexto y han desarrollado su labor en la región. Por su parte, la validación del instrumento de preprueba y posprueba por medio de alfa de Cronbach se vincula de manera directa con el cuestionario dirigido al total de la muestra; se realizó la prueba piloto con tres grupos de diez estudiantes. Es relevante aclarar que el valor de este índice "puede tomar valores entre 0 y 1 , donde 0 significa confiabilidad nula y 1 representa confiabilidad total" (Corral, 2009, p. 241); si su valor supera el 0,7 o el 0,8 existe fiabilidad, si el valor es menor, el instrumento se considera inconsistente.

Existen dos formas de encontrar este índice de consistencia interna: por medio de la varianza de los ítems y mediante una matriz de correlación. Se eligió el primer método. Para tal fin se utilizó el IBM sPss Statistics 22, "una familia de avanzados programas computacionales de análisis estadístico" (Méndez y Cuevas, 2014, p. 4), empleando ítems principales vinculados a dimensiones de variables. La medición se presentó con base en el diseño de escala Likert con cinco opciones de respuesta.

En este orden de ideas, el número de ítems, de varianzas individuales, la sumatoria de esas varianzas y la varianza del total los calculó el software para los treinta casos de la prueba piloto, con ayuda de una hoja de cálculo de Microsoft Excel. Se aplicó la fórmula del método de varianza, y se obtuvo el índice de $\alpha=0,7766$. Este valor ratificó la validez interna del instrumento.

\section{Técnicas y métodos}

\section{Para el análisis cuantitativo}

De manera puntual, el test de estilos de aprendizaje se aplicó en el 2014, la encuesta de caracterización de padres de familia y contexto familiar en febrero del 2015, y la preprueba - el instrumento de medición más importante- se aplicó en los dos grupos 
en febrero del 2015; ese mismo instrumento, como posprueba, se aplicó al finalizar el experimento en noviembre del mismo año. De ahí que en el análisis de resultados se evidencien cuatro gráficos por pregunta (véase la figura 1). La tercera encuesta la diligenciaron los docentes de media técnica en febrero del 2015.

\section{Para el análisis cualitativo}

El material audiovisual y textual recolectado se clasificó por dimensiones, subtemas, variables y categorización de indicadores. Este análisis no se limita a descripciones narrativas de los fenómenos (Hernández et al., 2014), sino que la descripción ofrecida proporciona un entendimiento profundo tanto al investigador como al lector. Para el análisis cualitativo de datos se manejaron los tiempos de la siguiente manera.

- Observación participante holística: un formato por grupo por cada mes del estudio-marzo a octubre del 2015, sin tener en cuenta junio ni julio por tiempos de vacaciones escolares-, 12 formatos en total, seis para grupo experimental y seis para grupo de control.
- Aplicación de prueba de salida en octubre del 2015 (muy importante para la discusión con 50 ítems).

- Sesiones de profundidad semiestructuradas o grupos de enfoque, esto es, las sesiones 1, 2 y 3 dirigidas a estudiantes, en dos momentos: antes y después de la aplicación de la metodología en el grupo experimental.

- Sesión 4, dirigida a docentes (se suprimió, debido a que la encuesta inicial ofreció información suficiente para la investigación por parte de los docentes).

- Sesión 5, dirigida a padres de familia en el 2015.

- En noviembre del 2015 se aplicaron las pruebas de salida (cuestionario principal) debido al diseño de preprueba, posprueba y control a los dos grupos de estudiantes que hacen parte de la muestra.

- Las conclusiones del análisis del enfoque mixto de la investigación se complementaron y surgieron al realizar procesos de triangulación o comparación entre los datos obtenidos.

7. Suponiendo que tienes clase en 10 asignaturas distintas durante una semana normal, ¿en cuántas realmente se emplea tecnología para apoyar las clases por parte del docente?

GR. CONTROL ENTRADA

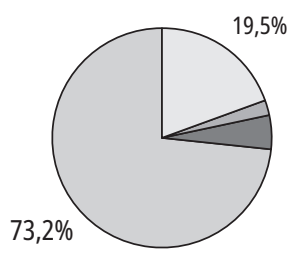

GR. CONTROL SALIDA

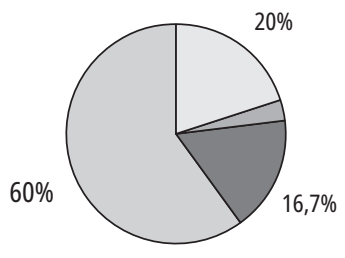

$\begin{array}{rr}0 \text { Asignaturas } & 16,7 \% \\ 2 \text { Asignaturas } & 60 \% \\ 4 \text { Asignaturas } & 20 \% \\ 6 \text { Asignaturas } & 3,3 \% \\ 8 \text { Asignaturas } & 0 \% \\ \text { Todas } & 0 \%\end{array}$

GR. EXPERIMENTAL SALIDA

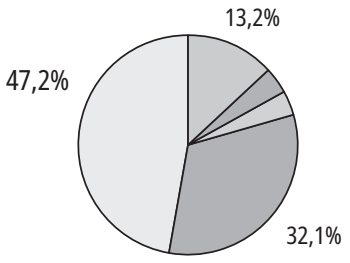

$\begin{array}{rr}0 \text { Asignaturas } & 0 \% \\ 2 \text { Asignaturas } & 32,1 \% \\ 4 \text { Asignaturas } & 47,2 \% \\ 6 \text { Asignaturas } & 13,2 \% \\ 8 \text { Asignaturas } & 3,8 \% \\ \text { Todas } & 3,8 \%\end{array}$ 2 Asignaturas $66,7 \%$ 4 Asignaturas $26,7 \%$ 6 Asignaturas $\quad 2,2 \%$ 8 Asignaturas $\quad 2,2 \%$ Todas $2,2 \%$

Figura 1. Ejemplo de análisis de preprueba y posprueba para cada pregunta. Fuente: elaboración propia. 


\section{Procesamiento de datos}

A fin de simplificar el análisis de datos y comprender la relación de indicadores que conforman cada una de las dimensiones de las dos variables con los ítems de instrumentos se generó la codificación que se presenta en la figura 2.

En otras palabras, al realizar el análisis de resultados de cada uno de los ítems en los instrumentos principales, se mencionan los códigos descritos, con el fin de, en primera instancia, vincularlos a los indicadores, a las dimensiones y a las variables de investigación, así como de sintetizar los gráficos estadísticos y los resultados. En segundo lugar, favorecen la comprensión y la interpretación de las conclusiones.

Para los instrumentos cuantitativos se realizó un análisis de porcentajes; dicho de otro modo, los datos originales obtenidos se transformaron en valores o símbolos a fin de que fuera posible tabularlos y contarlos. De ahí que, a fin de elaborar los gráficos estadísticos, las bases de datos por ítem, los cálculos y las sumatorias se emplearon formularios de Google (versión 2016), Microsoft Excel y el software IBM SPSs Statistics 22.

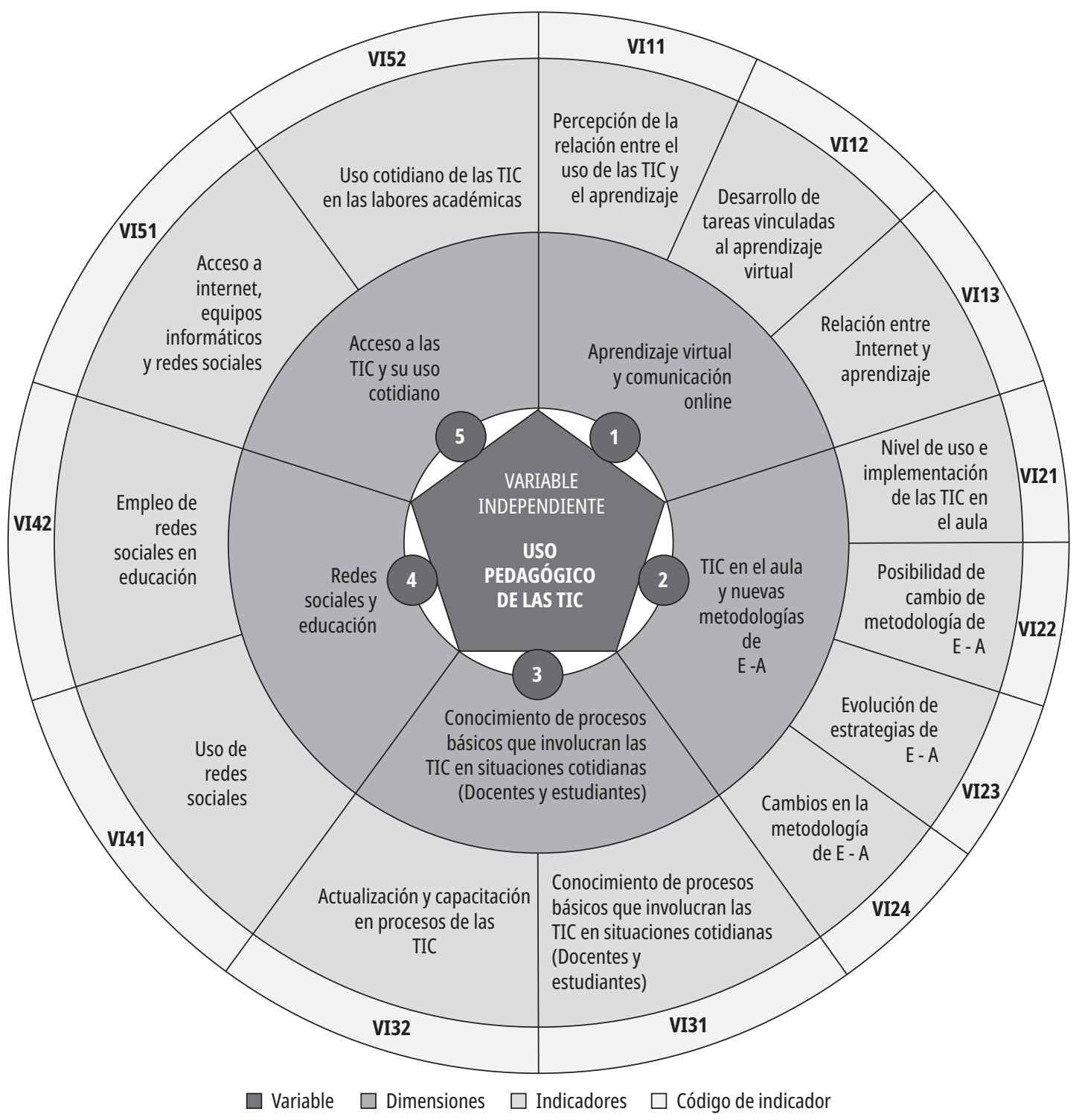

Figura 2. Codificación de indicadores: variable independiente. Fuente: elaboración propia. 


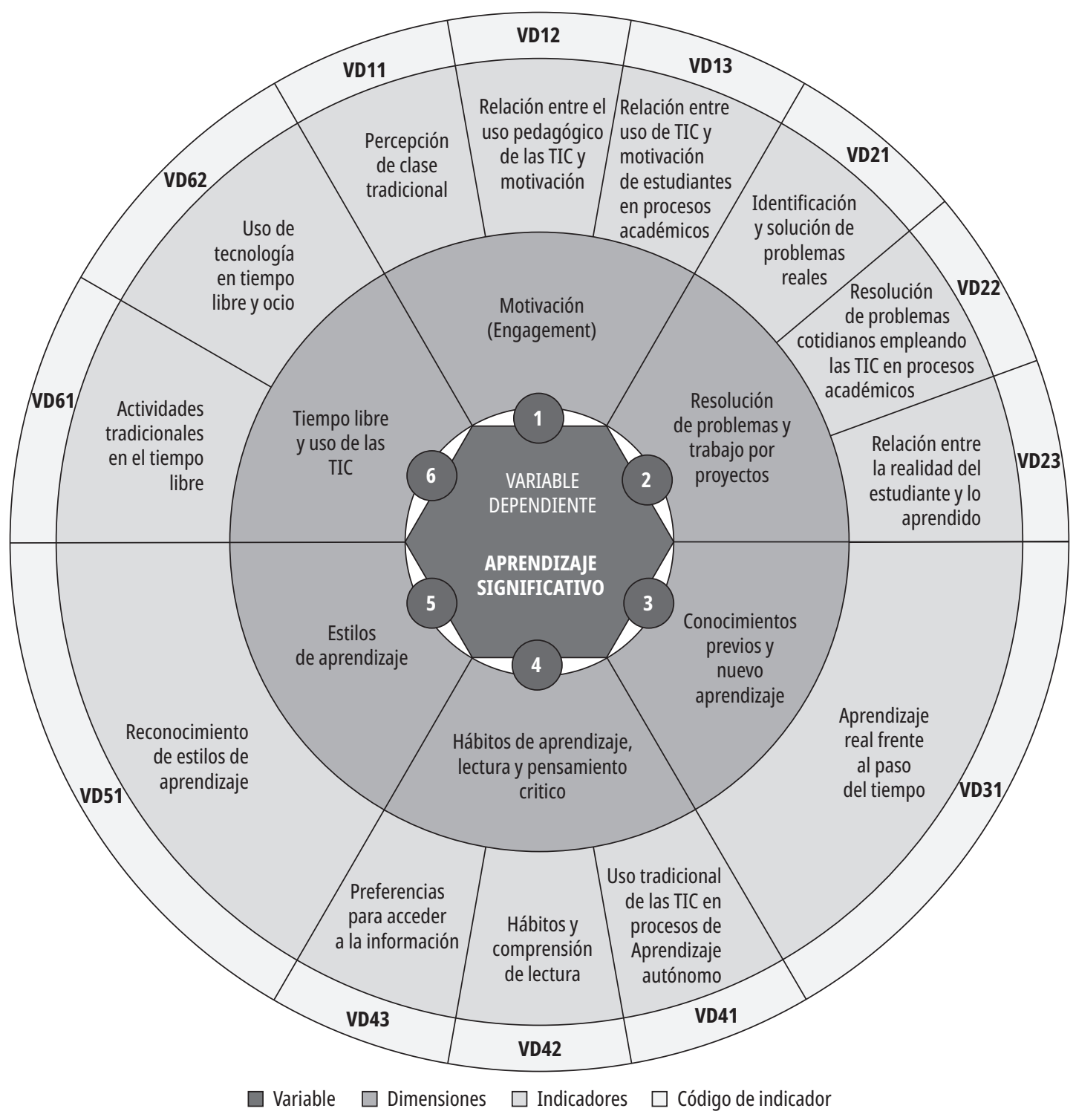

Figura 3. Codificación de indicadores: variable dependiente. Fuente: elaboración propia.

Al depurar los datos se buscaba encontrar inconsistencias tales como registros duplicados e incorrectos o información que no fuera relevante para la investigación. Esa información se identificó porque no tenía relación con los indicadores de cada dimensión y, en consecuencia, no aportaba al desarrollo del análisis. Luego, con los datos filtrados se acudió a la fase de eliminar los datos atípicos; en otras palabras, se suprimieron observaciones que en términos numéricos están más alejadas de la mayoría de los datos. La razón se resume en que podían generar engaños. En tercer lugar, se procedió a la selección de pruebas estadísticas, en la cuales se establecieron datos tales como la media, la moda y la desviación de ser necesario. Luego se dedujeron las propiedades y las características de la población a partir de la muestra.

El todo se dividió en sus partes fundamentales; se analizó cada uno de los resultados de las preguntas a fin de estar en capacidad de llegar a la fase de interpretación, en la que no solo se muestran números y gráficos, sino que se describe la relación de cada aspecto con los indicadores de las dimensiones. 
En cuanto a lo cualitativo, el proceso se desarrolló en tres etapas: reducción, disposición y transformación de información e interpretación. Este apartado culmina con el cronograma de tiempos estimados y ajustados para trabajo de campo y desarrollo de tesis doctoral. Luego, se identifica el presupuesto requerido para llevar a la práctica lo planteado; el total fue de 4579500 pesos colombianos en productos y de 3680000 en servicios.

Lo anterior sirve de preludio al capítulo que posibilita la identificación de datos y estadísticas de cada técnica empleada, para luego vincularlos a las conclusiones, con base en los objetivos planteados en la fase inicial de la investigación. El objetivo principal del procesamiento se resumió en inspeccionar, seleccionar y transformar los datos obtenidos, con la finalidad de resaltar información útil, todo con el propósito de generar conclusiones y tomar decisiones.

\section{Discusión y análisis de resultados}

\section{Sobre estilos de aprendizaje de estudiantes}

Se presentaron condiciones de equilibrio (entre el $30 \%$ y el $74 \%$ de los estudiantes). Esto sugería que los docentes, con el fin de fortalecer las dimensiones de aprendizaje, debían alternar distintas metodologías; en otras palabras, de continuar con la clase tradicional se desconocerían los resultados del presente estudio. Sin embargo, es importante resaltar que estos grupos también presentaron en menor medida "preferencias moderadas" (entre el $2 \%$ y el $38 \%$ de los estudiantes) y "preferencias fuertes” (entre el 2\% y el 33\%). Al realizar un análisis más detallado se observó que la dimensión "Visual/verbal" se encontraba dividida entre "más visual”, "mucho más visual” y “equilibrio”. En términos generales, entre las preferencias moderadas sobresalieron estilos más activos, más sensoriales, más visuales y más secuenciales. En "preferencias fuertes" se resaltaron estilos mucho más visuales en ambos grupos.
El estilo "mucho más visual" predominó a pesar del equilibrio relativo. En este, los estudiantes aprenden con significado a través de imágenes, diagramas, gráficas y videos, o bien por medio de lecturas y palabras sonoras; se necesitan nuevas metodologías que involucren estos aspectos en los procesos de enseñanza de la población. Precisamente, el uso pedagógico de las Tic diseñado se ligó a esos ítems, al entenderlos como medio, no como fin.

\section{Sobre la caracterización de los padres de familia (contexto)}

El instrumento se distribuyó en cuatro secciones, las cuales recogieron información sobre la caracterización general de las familias, las opiniones sobre el uso de tecnología por parte de los estudiantes, los hábitos de estudio y, por último, sus perspectivas o intenciones vinculadas al futuro académico y laboral cercano a ellos.

Se dedujo que las más interesadas en la educación de los jóvenes son las madres, antes que los padres. Tanto hombres como mujeres representantes tenían un promedio de edad bastante bajo, y el 67\% convivían con su pareja (en matrimonio o unión libre). También se encontraron otros factores comunes, como, por ejemplo, el bajo nivel de escolaridad y un bajo poder adquisitivo (principalmente, entre uno y dos salarios mínimos legales vigentes colombianos).

Casi la totalidad de los padres de la muestra deseaban que sus hijos estudiaran antes de trabajar, pero eran conscientes de la necesidad de hacerlo de forma paralela para lograr sus metas profesionales. Frente al uso de tecnología por parte de los jóvenes que tenían a cargo se presentó una contradicción con otras preguntas de la encuesta y lo expuesto por ellos en el grupo de enfoque. A continuación, se relacionan los detalles.

En la encuesta, casi el 57\% manifestó creer conocer con claridad los sitios web y las redes sociales que frecuentaban los estudiantes. Ahora bien, si el 70\% de los padres se dedicaba exclusivamente a trabajar y otro pequeño porcentaje trabajaba y estudiaba, se dedujo que los estudiantes 
usaban este tipo de herramientas tecnológicas muchas veces cuando los padres no se encontraban en el hogar.

En segunda instancia, el grupo de enfoque casi por unanimidad- expresó que conocía muy poco sobre los sitios web y las redes sociales frecuentados por sus hijos, y que una posible causa era el desconocimiento del tema. Además, muchos de los padres creían conocer los riesgos que involucraba el mal uso de redes sociales (cerca de 86\%), pero al manifestar el desconocimiento mencionado quedaron algunas dudas al respecto.

El concepto general de los padres frente al uso de internet y las redes sociales presentado por los estudiantes fue negativo. Afirmaron que perdían mucho tiempo en este tipo de herramientas y que su mal uso afectaba notoriamente su desempeño académico (el 93\% lo señaló). Asimismo, argumentaron que si se empleaban bien estas aplicaciones y los dispositivos asociados, era factible mejorar el nivel académico y, en consecuencia, su aprendizaje (el $81 \%$ así lo manifestó).

Expresaron también con respecto a las clases que la metodología clásica o tradicional sí debía cambiar, con el propósito de, en primera instancia, aumentar la motivación y el interés de los estudiantes al involucrar la tecnología; en segundo lugar, a fin de presentar distintas soluciones a problemas cotidianos por medio de lo que se aprende. De ahí que algo muy importante - y que no ameritaba discusión para ellos- era cómo el uso de las nuevas tecnologías de la información y la comunicación mejorarían el aprendizaje (el 95\% defendió esta posición).

También reconocieron la importancia de tener servicio de internet en sus hogares, y afirmaron que ha evolucionado el mundo y la forma de comunicarse. De ahí que los padres mostraron un gran interés por recibir capacitación de forma constante en el uso y la solución de problemas empleando la tecnología, al igual que sus hijos.

En los hábitos de estudio se presentó la segunda contradicción entre la encuesta y la información obtenida en la sesión grupal. En la primera, el 80\% de los padres esgrimieron que los jóvenes contaban con buenos hábitos de estudio, mientras que en el focus group muchos expresaron que no era así. Declararon leer muy poco por iniciativa propia y complementaron su idea al afirmar que no tenían verdaderos hábitos que potenciaran su nivel de lectura, escritura y habilidades matemáticas; apenas tres padres expresaron lo contrario y que sus hijos sí tenían claro su proyecto de vida.

\section{Sobre el uso de la tecnología por parte de los docentes}

Esta reflexión e interpretación de los datos no pretendió juzgar a los docentes por las dificultades metodológicas presentadas en la institución. En primera instancia, según el 55\% de los maestros las clases solo algunas veces eran agradables para los estudiantes, y el 30\% puntualizó que casi siempre. Dato contradictorio con la opinión de los estudiantes, tanto en la encuesta como en las sesiones de grupos de enfoque (focus group). Más adelante se registra la discusión de estos dos instrumentos, en el que causó sorpresa la contradicción entre docentes y estudiantes frente a la percepción de las clases.

De la misma forma, con respecto a la resolución de problemas reales con base en lo aprendido en el aula, la mayoría de docentes detallaron que siempre, casi siempre y algunas veces se da esta situación en los procesos académicos; las opciones "nunca" y "casi nunca", prácticamente, no se seleccionaron. La tendencia se mantuvo en los mismos niveles al preguntarles si promovían el gusto por la lectura y la escritura adentro y afuera del aula.

Por otra parte, el 95\% especificó que casi siempre y algunas veces los estudiantes aprendían cosas importantes y significativas para ellos con el sistema de clases que llevaban a cabo, pero fueron reflexivos al aseverar que las clases sí debían cambiar de alguna forma; el 100\% confirmó este enunciado. Al preguntar las razones, manifestaron que sentían cansancio de parte de los estudiantes por lo tradicional, y que las metodologías debían evolucionar de forma paralela a los cambios que se estaban presentando en la sociedad actual del conocimiento (entre otras razones).

Los docentes estaban convencidos de que el uso pedagógico de las TIC ayudaría a mejorar el nivel de aprendizaje significativo de los estudiantes; el 
85\% señaló que esta situación se presentaría siempre o casi siempre y, como consecuencia, este uso incrementaría el interés por la lectura y la escritura (el $80 \%$ así lo aseguró).

Según ellos, en las instituciones educativas públicas de la región, la falta de equipos, la baja calidad de los existentes, las dificultades de conectividad, la falta de conocimiento en el uso de herramientas y los nuevos modelos pedagógicos disruptivos eran aspectos constantes que limitaban el uso de la tecnología en procesos académicos con los estudiantes. A pesar de todos esos factores, el 95\% de los docentes utilizó la tecnología en clase de alguna forma al menos una vez durante el 2014 y el 2015.

Por otra parte, los datos recuperados sobre hábitos de los docentes en su tiempo libre se adaptaron al imaginario del investigador antes de aplicar la prueba. Veían en promedio televisión de una a tres horas al día; el 100\% usaba internet en su residencia entre dos y seis horas en ese mismo lapso y empleaba este servicio, principalmente, en labores académicas, de lectura, de acceso a plataformas de videos, de revisión de perfiles en redes sociales y aprendizaje online.

En lo que correspondía a las redes sociales, el 90\% de los docentes contaba con perfil en Facebook y el $72 \%$ ingresaba al menos una vez al día a revisar actualizaciones. Agregaron que las redes sociales bien empleadas eran herramientas útiles en el aprendizaje significativo de los estudiantes: el $35 \%$ expresó que "siempre", el 40\% "casi siempre" y el 25\% "algunas veces". De la misma manera, estaban de acuerdo en que el uso de dispositivos móviles en labores académicas permitiría dinamizar estrategias de enseñanza aprendizaje; en fin, realizó ese tipo de tareas y lo ratificó el 95\%.

Al tener en cuenta que el 100\% empleaba internet para aprender y el $80 \%$ utilizaba herramientas online para tareas específicas, pudo argumentarse que sí fue posible generar cambios metodológicos en clase por medio del uso de tecnología. Su voluntad se reflejó al evidenciar que el 100\% quería que las TIC se involucraran de alguna manera en sus clases; el 95\% de ellos deseaba recibir formación al respecto.

\section{Sobre la percepción de modelos de clases por parte de estudiantes y el diagnóstico de uso cotidiano de la tecnología (preprueba y posprueba para los dos grupos)}

A fin de comprender esta interpretación de resultados, el lector debe remitirse a las figuras 2 y 3 para identificar indicadores, dimensiones y variables. Por ejemplo, el indicador vD11 corresponde al primer indicador de la primera dimensión de la variable dependiente (vD), denominado "Percepción de clase tradicional"; luego de esa aclaración, puede abordarse la síntesis del análisis global y particular.

La percepción de la forma como se dieron las clases en el grupo experimental mejoró notablemente; los estudiantes consideraron al finalizar el experimento que era mucho más agradable, y la primera pregunta mostró la variación en los dos grupos (“mejoró vD11”). Los estudiantes del grupo experimental en la prueba de salida expresaron que el nivel de solución de problemas en las clases aumentó ("mejoró vD21"). De la misma forma, el número de asignaturas en las que se presentaba interés por el aprendizaje y la aplicación de lo aprendido en la vida diaria subió luego de aplicar el experimento ("mejoró vD12 y vD13").

La lectura por iniciativa propia se incrementó sutilmente en la posprueba del grupo experimental y este ítem mantuvo su nivel en los dos grupos; en otras palabras, el experimento no influyó de forma significativa en iniciativa de lectura, ni en la percepción de la motivación por parte de las distintas asignaturas en este aspecto ("leve tendencia a incremento en: vD42, vD41, vD11, vD42”).

La percepción de la frecuencia con la que aprendían cosas significativas e importantes aumentó en el grupo experimental, principalmente, en los índices de "casi siempre". De forma opuesta, "casi nunca" disminuyó ostensiblemente ("mejoró vD31 y vD23"). Con respecto a la percepción de la cantidad de materias que usaban tecnología, el grupo experimental se diferenció radicalmente en el momento de salida; los índices también 
aumentaron, sobre todo, en los valores de cuatro y seis asignaturas ("mejoró vi52”).

Prácticamente, la totalidad de los estudiantes en los dos grupos aseguraron la necesidad de cambio de la metodología de clase tradicional ("estable con máximos niveles de aprobación: vD11 y vi22”). Manifestaron de forma explícita que si las clases fueran como en Diseño Publicitario Empresarial y Tecnología e Informática - las asignaturas del experimento- mejorarían su motivación e interés por aprender, y que la rutina de las otras clases y la falta de recursos los desmotivaba y no permitía un verdadero aprendizaje (la segunda parte se dedujo con respuestas de pregunta abierta).

Los estudiantes de los dos grupos en los dos momentos revelaron que la tecnología sí ayudaba a mejorar la forma como ellos aprendían en clase y fuera de ella ("estables con altos valores: vD13, vi11 y vi23"). Se infirió, además, al analizar los datos, que el empleo de tecnología en el aula optimizaba su aprendizaje, luego de que el valor máximo apareciera en la posprueba del grupo experimental con $54,7 \%$ en la pregunta 10 ("leve tendencia a incremento, con valores altos de salida en: vD13, vD31, Vi11, vi22, vi52"). Este tipo de conclusiones se complementaron con las obtenidas en la pregunta 11, y el resultado muestra que el uso de la tecnología en las clases del grupo experimental fue muy superior al grupo de control ("mejoró vi52, vi21, vD11").

Los mayores porcentajes de uso de tecnología fueron para el grupo experimental de salida (100\% en las dos asignaturas del experimento), contabilidad $(67,9 \%)$, inglés $(60,4 \%)$ y física $(60,9 \%)$. Los valores más bajos correspondieron a ciencias políticas y educación física (0\%), visión empresarial y castellano (15,1\%) y filosofía (22,6\%). Al comparar, pudo resaltarse que Tecnología e Informática y Diseño Publicitario Empresarial lideraron en términos de opinión y percepción ("mejoró vi52 y VI21").

En los dos momentos, para los dos grupos, el gusto, el engagement ("el compromiso") y la motivación se incrementan si se usan de manera pedagógica las TIC en las metodologías de enseñanza aprendizaje ("estables con máximos valores: vD12, vD13, vi23”).
La percepción sobre el dominio de tecnología por parte de los docentes no fue buena en el grupo de control, mientras que el experimental tuvo una mejoría en la percepción de este tipo de habilidades ("mejoró levemente vi31"). Del mismo modo, los estudiantes del grupo experimental estaban seguros de manejar mejor la tecnología que sus maestros (vi31, vi52), manifestaron con respuestas abiertas su bajo nivel de uso de tecnología en clase y formularon posibles causas, las cuales se resumen en falta de formación o recursos y miedo por innovar.

Para el grupo de control, usar tecnología en clase sí mejoraba el nivel de lectura y escritura de los estudiantes, mientras que el grupo experimental no tuvo cambios significativos. Claro está que al finalizar el experimento subió levemente el nivel de lectura en el grupo experimental ("incremento leve en: vD41, vD42, vD43").

Al terminar el proceso casi la totalidad de estudiantes del grupo experimental tenía computador en casa; esta cifra en el grupo de control fue del $80 \%$. El 60\% del grupo de control salida (GCs) tenía servicio de internet, mientras en el grupo experimental salida (GES) era el 89\%; el uso pedagógico de Tic influyó al reconocer la importancia del servicio en los hogares de la muestra, y se reportó un uso promedio diario de entre dos y seis horas (“mejoró vi51, vi52, vi13”).

Los smartphones fueron los principales dispositivos de acceso a internet - valor máximo de 66\% en GES-, seguidos por computadores portátiles y equipos de escritorio ("leve incremento en VD43, vi51, vi62"). Los hábitos de acceso a internet cambiaron significativamente al aplicar el experimento y se evidenciaron diferencias frente al grupo de control. Los incrementos se centraron en consumo de videos online, aprendizaje online, acceso a redes sociales y lectura ("mejoró vD42, vD41, vi41, VI13").

En cuanto a redes sociales, el 100\% de los estudiantes del GES contaba con perfil activo en Facebook (red empleada como aula virtual en el proyecto pedagógico). El GE, luego de haber empleado las redes sociales en su proceso pedagógico durante el 2015, mejoró la percepción de la relación entre buen uso y aprendizaje. Entre el 62\% 
y el $66 \%$ de los estudiantes accedía varias veces al día a estas de herramientas tanto para revisar contenidos y material multimedia publicados como para actividades de índole personal ("mejoró vi12, VI42, vi21, vi21, vi41, vi51, estable vD62).

Debido a actividades académicas ligadas a las redes sociales, se apreció un gran incremento en la creación y el uso de herramientas tales como Twitter, Pinterest, LinkedIn, Instagram, YouTube y Spotify. Ahora bien, los valores de Ges fueron: Twitter 100\%, Pinterest 94,3\%, LinkedIn 18,9\%, Instagram $77,4 \%$, YouTube $92,5 \%$, Spotify $56,6 \%$, y WhatsApp 86,8\%. La relación entre el GCS y el GES es bastante cercana a la presente entre el grupo experimental entrada (GEE) y el GES. El experimento sí influyó en el manejo de redes sociales virtuales distintas a Facebook en el grupo experimental de forma notoria ("mejoró vD62, vD61, vi51, vi52, vi41, vi42”). Expresaron, además, que tenían conocimientos sobre el posible uso de dispositivos móviles en clase para dinamizar procesos pedagógicos y mejorar así su aprendizaje por medio del uso de TIC; del mismo modo, se incrementó la cantidad de estudiantes que poseía ese tipo de instrumentos en el GE ("Mejoró vi51, vD62").

Los usos de dispositivos móviles en el GC se basaban en el entretenimiento. Por otra parte, el grupo experimental mejoró su uso en términos de aprendizaje online y offline, lectura (levemente), edición de documentos y uso responsable de redes sociales como, por ejemplo, aulas virtuales y materiales digitales de consulta ("mejoró vi42, vi41, VI13, VI11, VI12, VI21, VD43, VD42, VD41, VD62, vD61, vD13”). Además, el 81,1\% del GEs sí leyó libros, revistas, documentos, tomaron apuntes o desarrollaron alguna actividad de orden académico en smartphones o tablets durante el experimento ("mejoró vi52, vi12, vi21").

Al finalizar, se presentó un incremento del $20,5 \%$, con valor de salida del $84,9 \%$. La forma en la que vincularon internet con aprendizaje se acrecentó notoriamente ("mejoró vi13, vi24, vi52, VI12, VD62, VD41, VD12”). Sustentaron con respuestas abiertas ejemplos de lo que aprendieron por medio de videos, cursos e investigación personal (“mejoró: vD23, vD22, vD21, vi13).
Existía una tendencia clara a aprender con videos interactivos y dinámicos desde internet y material multimedia, como, por ejemplo, animaciones, sitios web e infografías. Un porcentaje considerable sosntenía que al unir los tipos de aprendizaje planteados en las opciones de respuesta podrían darse buenos resultados. El GC mantuvo sus resultados en los dos momentos; en el GE el uso de videos interactivos y dinámicos desde internet y material multimedia para el aprendizaje se incrementó (flipped learning), pero sorprendió que la frecuencia de lectura en formato digital prácticamente no se haya modificado ("VI11, VI52, VI24, VD11, VD12, VD41, VD22").

De ahí que prácticamente el $100 \%$ de los dos grupos en los dos momentos evidenciaran unanimidad; los estudiantes estaban de acuerdo con que las clases de las diferentes asignaturas en la institución emplearan tecnología para aprender más y mejor ("constantes con máximo valor: VI22, VI23, VD12”). Con respecto a la relación entre motivación, dinamismo, lúdica y aprendizaje para los estudiantes de los dos grupos, en los dos momentos, existía una relación directa entre estos aspectos ("estables con alto nivel VD31, VD11, VI12"). De ahí que casi el 100\% estuviera de acuerdo con que el uso pedagógico de las TIC aumentaba su motivación por aprender ("VD11, VD13, VD31, VI52, VI11, VD51, VI23"). Al respecto, prácticamente la totalidad de la muestra expresó su interés en recibir formación periódica en el uso de TIC para acceder al conocimiento y potenciar su aprendizaje ("estable con el máximo valor VI31, VI32").

En conclusión, los estudiantes manifestaron que a pesar de abordarse teorías en las clases, no se ligaban con su contexto; en consecuencia, no había significado para el aprendizaje, debido a que se convertía en un ejercicio mecánico sin trascendencia. El 83\% del GEs afirmó, al terminar, que resolver problemas de la vida cotidiana en clase aumentaría su nivel de aprendizaje ("mejoró vD21, VD22, VD11"). Los estudiantes, al igual que los docentes y los padres de familia anhelaban cambios metodológicos en las estrategias de enseñanza y aprendizaje en la población, y de forma explícita declararon que el uso pedagógico de las Tic era 
necesario para mejorar el aprendizaje significativo en todas las asignaturas.

\section{Observación participante holística. Grupo de control. Sesiones 1, 3, 5, 7, 9 y 11}

Para empezar, se hacen las siguientes aclaraciones: en las sesiones 11 y 12 se aplicó la prueba de aprendizaje de salida para los dos grupos; en las demás se manejaron los mismos contenidos de forma paralela (sesión 1 con 2, 3 con 4, 5 con 6, etc.), la diferencia fue siempre la metodología, tal como se explicó.

Se encontraron patrones de comportamiento por parte del grupo de control en las sesiones observadas, entre otros, la motivación y el interés con alto nivel solo durante los primeros minutos, a pesar de la intención de dinamizar las clases y de generar interés por parte del docente.

En la Sesión 1 hubo poco interés por el tema en la mayoría de los estudiantes; cerca de cinco personas siguieron indicaciones tal como se explicó. Luego de 20 minutos, se presentaron reacciones de agotamiento en las actividades propuestas.

En la Sesión 3 se presentaron reacciones positivas en la fase de preguntas inicial por parte del docente, al vincular la realidad del estudiante con el tema. El interés bajó luego de 15 minutos de exposición del profesor. La interacción en la fase 3 y 4 mejoró un poco la motivación de los estudiantes. La exposición grupal final se distinguió por la falta de atención de los compañeros (se notaban cansados). En la reflexión grupal se generó debate, y en el trabajo por empresas —énfasis de la media técnica- se vio trabajo en equipo en busca del bien común. En las exposiciones se notaron tímidos. Las dudas se resolvieron de forma personalizada durante la explicación magistral y en la sustentación frente a los compañeros. Hubo mayor participación con respecto a la Sesión 1, pero el patrón de comportamiento fue evidente.

En la Sesión 5 la atención y la motivación durante 15 minutos disminuyó al trabajar con copias y al realizar el listado. Un ejercicio de conversión de unidades de medición ayudó a la participación, pero no lo que se esperaba. Para la fase final, la atención se había perdido en cerca de la mitad de los estudiantes, lo cual influyó en los demás. El trabajo colaborativo solo se presentó en la fase del ejercicio matemático; hubo interacción y trabajo colaborativo en grupos de tres personas. Desde la presentación magistral y en las etapas 2, 4 y 5 se solucionaron dudas de estudiantes (muy pocas).

La Sesión 7 presentó bajo nivel de atención y motivación después de los diez minutos iniciales, pues los estudiantes se distraían fácilmente. En las otras fases hubo apatía por la clase, y solo en la actividad final subió el interés del grupo. El trabajo colaborativo se dio en la lectura por parejas y en la actividad final. El bajo nivel de motivación e interés condujo a que los estudiantes no preguntaran e interactuaran como se esperaba en el diseño de la clase. Una vez más las preguntas surgieron en la fase final; al parecer los conceptos no fueron apropiados de la mejor manera.

En la Sesión 9, en la Fase 2, se mantuvo el nivel inicial de motivación, pero la exposición con diapositivas lo cambió. Esta situación continuó hasta el final a pesar de la buena disposición del docente. De forma similar, el trabajo colaborativo no fue el mejor, pues en la reflexión grupal hubo bastante interacción y debate sobre los beneficios del tema en su cotidianidad (emarketing para empresas). Se concluyó que la reflexión grupal permitió elevar el nivel de interés por los temas a tratar, y que las lecturas y la exposición magistral no fueron del total agrado de los estudiantes.

\section{Observación participante holística. Grupo experimental. Sesiones 2, 4, 6, 8, 10, 12}

En la Sesión 2 se mantuvo el interés durante toda la actividad, evidente en la relación de contenidos con la realidad y los conocimientos previos durante las dos horas. De forma permanente se cuestionaron unos a otros en la fase de ideas previas. El trabajo colaborativo se presentó en la investigación y la construcción del concepto. Hubo telecolaboración para la presentación final. Muchas preguntas y participación en la investigación grupal y el uso de herramientas o equipos informáticos al ser la primera vez que los empleaban. 
En la Sesión 4 se vio un alto nivel de motivación, compañerismo y libertad para preguntar en las seis fases. Asimismo, manifestaron de forma libre la funcionalidad de la nueva herramienta en línea para crear infografías de calidad, de manera que podían expresar sus ideas de una forma diferente. El tablero de Pinterest se convirtió en un muro virtual para exponer sus ideas.

El trabajo colaborativo comenzó con el diseño de infografías, continuó con la investigación y el uso de Piktochart, y terminó con la publicación en el tablero telecolaborativo. Se generó controversia con el cuestionamiento propuesto en la Fase 1. También hubo acompañamiento constante para resolver dudas sobre el uso de la herramienta y orientar iniciativas de diseño. Durante la fase de investigación surgieron muchas dudas de contenido y presentación de infografías, las cuales se solucionaron a medida que se hacían evidentes. En síntesis, hubo una nueva perspectiva del tema con base en las opiniones de los compañeros en la investigación. Se terminó el diseño de la infografía en Pinterest. Hubo gran motivación al trabajar en el tablero virtual y compartir sus productos.

En la Sesión 6 continúa como factor común la motivación durante las siete fases (dos horas). Participación constante con aportes, experiencias y preguntas. Leve nivel de distracción durante la Fase 4. El trabajo colaborativo se evidenció en las fases 1, 3, 5, 6 y 7. La sinergia en todo el desarrollo de la clase fue fundamental para la construcción del conocimiento. Solo en las fases 2 y 4 no interactuaron con sus pares. Curiosidad y muchas preguntas sobre el tema, principalmente en fases 1, 3, 4 y 6; en muchas ocasiones, las dudas fueron resueltas por los compañeros. En resumen: interés constante y un buen nivel de motivación por el uso de herramientas tecnológicas. Se concluye que los videos deben ser cortos para no perder la atención de los estudiantes.

En las sesiones 8 y 10 se visualizan comportamientos similares. Desde la Fase 1 fue posible apreciar interés en el tema y la ansiedad por trabajar en su propio e-book, al saber, por supuesto, que beneficiaría a cada una de las empresas. El trabajo fue dinámico y agradable para todos. La comunicación grupal fue evidente. Desde la Fase
2 mostraron alta motivación aprendiendo e investigando; el docente agregó música electrónica instrumental de fondo en las fases de investigación, publicación y diseño. Lo anterior, al parecer, permitió cierto grado de libertad para resolver dudas y brindar confianza en las dudas que, por cierto, fueron principalmente de procedimientos y no de contenido.

La motivación y el interés de la penúltima clase observada fue alta en todas las fases. Lamentablemente, en el segundo video se notaron muestras de cansancio (videos de más de seis o siete minutos definitivamente no son convenientes). Las fases finales generaron buen ambiente, fundamentado en aprendizaje colaborativo y creatividad. El trabajo colaborativo se dio en las fases 4 , 5,6 y 7, principalmente, y permitió un alto nivel de producción intelectual. Aproximadamente un $70 \%$ de los estudiantes del grupo experimental expresaron sus inquietudes y dudas en el transcurso de las siete fases en las dos horas. Las dudas, principalmente, fueron de contenido y manejo de la nueva aplicación móvil utilizada en el proceso (TAWE). Se apropiaron los contenidos y se desarrollaron habilidades y destrezas de trabajo en equipo y adquisición de nuevas herramientas empleadas como medio para el aprendizaje.

\section{Grupos de enfoque (focus group) con estudiantes (tres sesiones, dos momentos)}

En la Sesión 1 se encontró que para los estudiantes no era agradable la forma como se impartían las clases en la institución. Los docentes solo empleaban la clase magistral, en consecuencia, se cansaban muy rápido y no se lograban los objetivos de aprendizaje. Había mucha teoría y poca práctica; proponían cambios por medio de juegos, dinámicas innovadoras, con actividades en las que todos aportaran con orientación del docente. Las clases se limitaban, en muchas ocasiones, simplemente a observar, escribir y hablar cuando el docente lo solicitaba; según ellos, se basaban en la memoria y la repetición de conceptos, y ponían en tela de juicio su comprensión. 
Los resultados coincidieron con los instrumentos cuantitativos al evidenciarse que solo en algunas ocasiones los estudiantes sentían que estaban aprendiendo con agrado. También eran conscientes de las pocas herramientas que los docentes tenían a su alcance y los recursos de la institución les resultaban bastante limitados; sostuvieron que eso también desmotivaba a los educadores. Además, de forma unánime expresaron su intención de transformación de las clases, manifestaron que esperaban cosas nuevas y, en parte, que el rechazo se presentaba debido a la desactualización tanto en metodologías como en contenidos. También afirmaron que se seguía con la misma forma de enseñar año tras año y eso los desmotivaba. No obstante, aclararon que los cambios no debían ser totales, ya que hay cosas buenas por rescatar de cada metodología.

En una síntesis de los resultados, expresaron que el aprendizaje de las pocas cosas que permanecían a través del tiempo en ellos se vinculaba, de forma directa, con el interés suscitado, es decir, aprendían lo que verdaderamente les llamaba la atención. Complementaron que al parecer el fin era solo aprobar, no para que el aprendizaje trascendiera y se pudiera aplicar. Lo que se veía en clases - según lo que expresaron- muy pocas veces se aplicaba y se tomaba más como datos de cultura general, porque no se empleaba en resolver problemas de su realidad.

Según ellos, en las clases no se motivaba para la lectura y la escritura. No les gustaba leer porque no tenían el hábito y sentían apatía por practicar. Preferían usar dispositivos móviles por lapsos extensos. Además, entre otras de sus apreciaciones afirmaron que no escribían bien porque no se sentían inspirados para hacerlo. También cómo la cultura de poco esfuerzo en sus hogares influía, así como el uso de tecnología; preferían ver videos, chatear o estar en redes antes que leer (aspecto que concuerda con los resultados del test de estilos de aprendizaje). Finalmente, concluyeron que sus problemas de lectura, escritura y lógica matemática sí influían de forma directa en su desempeño académico.

Les gustaría tener aulas especializadas, cómodas y dotadas con tecnología. Por consenso expresaron que con menos estudiantes aprenderían más, sería mejor el ambiente, comprenderían mejor y los profesores tendrían más tiempo para cada uno de ellos. Pidieron menos clase magistral, ambientes agradables, conocimientos nuevos para la vida diaria y participación activa voluntaria al aprender; de ser así, expusieron que la participación sería masiva. Así se concluye el análisis y la discusión, luego de los dos momentos.

En la Sesión 2 sustentaron que emplear tecnología en el aula posibilitaba la existencia de nuevas formas de hacer las cosas; dado que permite encontrar más información que en la clase tradicional, deben existir metodologías especializadas en el uso de TIC, con docentes orientadores - no dictadores- a fin de que el estudiante construyera su conocimiento por medio de ejercicios didácticos. Se infirió, al escucharlos, que la tecnología educativa no solo debía enfocarse en usar herramientas de la web; hablaron de realidad virtual, aumentada y aplicaciones especializadas en temas y funciones particulares, y sobre cómo la tecnología ayuda a dinamizar y motivar procesos de desarrollo del aprendizaje. Los estudiantes creen que aprenderían más al emplear tecnología tanto en el aula como fuera de ella, y así aprender a resolver problemas cotidianos; complementaron sus ideas al afirmar que las Tic permitían cambiar el paradigma de ir solo a aprender por ir a descubrir.

También plantearon que la motivación y las ganas de aprender mejorarían mucho al emplear tecnología, pero con una metodología que se adapte a esta. El modelo de clases normalmente era rígido y no permitía que la tecnología se empleara; manifestaron que los jóvenes actuales aprenden de forma distinta. Casi de forma unánime expresaron al finalizar el estudio que empleaban tecnología precisamente en las asignaturas que hacían parte del experimento (tabletas, programas para crear contenidos digitales, edición de audio y video, etc.). Explicaban que en esas materias, el aprendizaje no se daba solo en el aula, sino que gran parte del aprendizaje era autónomo, de ahí que les llamara mucho la atención las clases por medio del canal del proyecto en YouTube (flipped learning).

Les gustaban esas clases porque, entre otras cosas, las redes sociales se utilizaron para aprender 
y aprovechar el tiempo, de modo que su uso cambió de manera sustancial. Esto fue positivo porque permitió que sus opiniones y creaciones se tuvieran en cuenta en esos escenarios. Según ellos, las posibilidades ofrecidas por la clase invertida eran múltiples, se interesaban más por los temas; antes no se empleamban esas herramientas porque se consideraba que no posibilitaban trabajo productivo, pero ahora se usan para aprender y descubrir.

Finalmente, los estudiantes esbozaron que en múltiples ocasiones los docentes no sabían usar la tecnología, decían que no les gustaba o que sentían algo de temor al utilizarla. En otros casos, notaban que los profesores no contaban con el conocimiento para dar una clase con nuevas herramientas y preferían seguir siendo tradicionales, sin permitir nuevas posibilidades para resultados diferentes de aprendizaje. Al finalizar la sesión y a modo de complemento, esgrimieron que como consecuencia del uso responsable de las TIC por parte de los docentes y los estudiantes sí se mejoraría el nivel de lectura y escritura, sin importar la asignatura.

En la Sesión 3, con respecto a los hábitos tradicionales, se resumen las siguientes conclusiones en la discusión: según los estudiantes se incrementó su nivel de lectura en el transcurso de la aplicación del experimento; debe recordarse que dicho incremento no fue notorio en el análisis cuantitativo. Afirmaron, además, que disminuyó la cantidad de horas de consumo de contenidos televisivos. Cerca del $80 \%$ tenía computador en casa, un $60 \%$ de ellos compartía su uso con una o dos personas más, para el $40 \%$ restante el uso es personal y cerca del $70 \%$ tenían servicio de internet en casa.

En la sesión de salida, el 90\% tenía smartphones y los usos principales eran: descargar aplicaciones (como, por ejemplo, juegos), resolver tareas, tomar apuntes, redes sociales, fotografías, edición de imágenes y lectura. Mientras que en el focus group de entrada seis personas de diez habían descargado aplicaciones, en la salida todos respondieron que lo habían hecho. Además, antes de la sesión de salida habían leído libros, artículos, publicaciones en redes o tareas, así como habían tomado apuntes en smartphones o tabletas, principalmente, en las asignaturas Diseño Publicitario Empresarial y Tecnología e Informática.

En resumen, los sitios web más visitados por el grupo de salida fueron redes sociales, lo que incluye a YouTube, con fines educativos y de entretenimiento, y páginas de noticias, académicas o libros. Los resultados se distancian del grupo de entrada.

Los estudiantes del grupo de salida declararon que habían empleado la web para aprender durante el último año y había servido la metodología de videos para ese fin, pues aprendieron de forma virtual y autónoma. En consecuencia, tenían una perspectiva distinta sobre el uso de internet y el empleo de la tecnología para aprender. Finalmente, señalaron que el cambio había sido radical $y$, definitivamente, tanto internet como la tecnología sí potenciaban de forma significativa el acto de aprender; les gustaría que se aplicaran metodologías que usen TIC en las clases (similar a los demás instrumentos).

\section{Sesiones de grupos de enfoque (focus group) con padres de familia}

Los padres no conocían el tipo de acciones que desarrollaban sus hijos con los distintos dispositivos electrónicos. Además, dieron a conocer que los estudiantes muchas veces aparentaban realizar compromisos académicos, pero realmente estaban en otras actividades. Según ellos, los jóvenes perdían mucho el tiempo en chats y redes sociales; los padres no se percataban del tipo de personas con las que se comunicaban, y agregaron que conocían muy poco sobre el tema.

Asimismo, dejaron claro que podían ser herramientas muy buenas para la comunicación inmediata en el trabajo o con la familia. Consideraban relevante tener computador e internet en casa; curiosamente, concretaron que sí los habían empleado para aprender (sobre recetas, consejos para el hogar y procesos administrativos generales); según ellos, este tipo de tecnologías es muy importante para la familia.

Para los integrantes del grupo, servicios de internet y redes sociales como YouTube resultaban ser útiles para aprender si se empleaban bien. 
Expresaban que al emplear este tipo de herramientas los procesos debían vigilarse, debido a que se corrían muchos riesgos. Frente a esto, se presentaba un gran inconveniente al no tener conocimiento sobre las acciones de los hijos en la web, y era difícil supervisarlos ya que los estudiantes eran muy ágiles en el uso de este tipo de aplicaciones y dispositivos.

Al aplicar la guía semiestructurada, también se evidenció que para ellos era muy importante que los estudiantes utilizaran tecnología para solucionar problemas de la vida diaria. Al respecto, complementaron al afirmar que la evolución tecnológica de los últimos años había influido de forma significativa en sus hábitos $\mathrm{y}$, prácticamente, no habían tenido otra opción sino aprender, muchas veces junto a sus propios hijos.

En cuanto a hábitos de estudio, lectura y escritura, se presentó una diferencia notoria entre el focus group y el cuestionario. La mayoría de los padres expuso que sus hijos no tenían verdaderos hábitos de estudio que propendieran a niveles diferentes de lectura, escritura y habilidades matemáticas. En segunda instancia, plantearon que de forma esporádica tenían iniciativa para acceder al conocimiento y explorar nuevos aprendizajes; lamentablemente, este tipo de acciones no se presentaban de manera constante.

Propusieron modificar el modelo de las clases en la institución; desde su perspectiva, los estudiantes no se interesaban en actividades académicas, la metodología tradicional no los motivaba y cumplían por obligación, por el objetivo macro de aprobar sin importar su nivel de aprendizaje. Finalmente, adicionaron que las clases eran rutinarias, monótonas y poco dinámicas; según ellos, eso iba en contra de un verdadero aprendizaje. Una posibilidad planteada por los padres para dinamizar estos procesos era utilizar tecnología, a fin de que los estudiantes aprendan más motivados y mejor.

\section{Sobre la prueba de conocimientos (dos grupos). Prueba de salida (octubre 2015)}

Este instrumento fue de vital importancia para redactar las conclusiones de la investigación. Se evidenciaron diferencias notables. Así, por ejemplo, fue superlativa la influencia del uso pedagógico de las TIC en el aprendizaje significativo de los estudiantes del GE; la metodología tuvo importancia medular durante el experimento. Es interesante analizar detalles de la información recolectada, tales como los que se presentan en la Tabla 3.

Al realizar el análisis, de la revisión se infiere que el $74 \%$ del GE superó al GC entre el 10,1\% y 49,7\% (diferencia máxima) en la prueba académica final, datos que por cierto sorprendieron al investigador por la considerable diferencia. En el documento final de la tesis doctoral se realiza el análisis de cada uno de los ítems. Asimismo, el GC superó al GE en el $8 \%$ del total de la prueba. La metodología propuesta, fundamentada en el uso pedagógico de las TIC, mostró resultados evidentes de aprendizaje al aplicar esta prueba.

Tabla 3. Detalles de las diferencias entre los grupos del experimento (prueba de salida)

\begin{tabular}{|c|c|c|c|c|c|c|c|c|c|c|c|}
\hline \multirow{2}{*}{\multicolumn{2}{|c|}{$\begin{array}{l}\text { Ítems en los que el } \\
\text { grupo control superó al } \\
\text { grupo experimental en } \\
\text { aciertos }\end{array}$}} & \multicolumn{10}{|c|}{ Ítems con diferencia a favor del grupo experimental en aciertos } \\
\hline & & \multicolumn{2}{|c|}{$\begin{array}{c}\text { Entre } \\
\text { oy } 10 \%\end{array}$} & \multicolumn{2}{|c|}{$\begin{array}{c}\text { Ente } \\
10,1 \text { y } 20 \%\end{array}$} & \multicolumn{2}{|c|}{$\begin{array}{c}\text { Entre } \\
20,1 \text { y } 30 \%\end{array}$} & \multicolumn{2}{|c|}{$\begin{array}{c}\text { Entre } \\
30,1 \text { y } 40 \%\end{array}$} & \multicolumn{2}{|c|}{$\begin{array}{c}\text { Entre } \\
40,1 \text { y } 50 \%\end{array}$} \\
\hline $\begin{array}{l}\text { Cantidad } \\
\text { de ítems }\end{array}$ & $\begin{array}{l}\% \text { del } \\
\text { total }\end{array}$ & $\begin{array}{l}\text { Cantidad } \\
\text { de ítems }\end{array}$ & $\begin{array}{l}\% \text { del } \\
\text { total }\end{array}$ & $\begin{array}{l}\text { Cantidad } \\
\text { de ítems }\end{array}$ & $\begin{array}{l}\% \text { del } \\
\text { total }\end{array}$ & $\begin{array}{l}\text { Cantidad } \\
\text { de ítems }\end{array}$ & $\begin{array}{l}\% \text { del } \\
\text { total }\end{array}$ & $\begin{array}{l}\text { Cantidad } \\
\text { de ítems }\end{array}$ & $\begin{array}{l}\% \text { del } \\
\text { total }\end{array}$ & $\begin{array}{l}\text { Cantidad } \\
\text { de ítems }\end{array}$ & $\begin{array}{l}\% \text { del } \\
\text { total }\end{array}$ \\
\hline 4 & $8 \%$ & 8 & $16 \%$ & 6 & $12 \%$ & 14 & $28 \%$ & 6 & $12 \%$ & 12 & $24 \%$ \\
\hline \multicolumn{2}{|c|}{$8 \%$} & \multicolumn{10}{|c|}{46 ítems = 92\% } \\
\hline
\end{tabular}

Fuente: elaboración propia. 


\section{Conclusiones y recomendaciones}

Esta parte se vincula de manera directa con los objetivos específicos, entendidos como sustento primordial del objetivo general y de la hipótesis. Se presenta entonces la síntesis de las conclusiones y las recomendaciones de este trabajo doctoral de casi cuatro años de duración.

\section{Objetivo específico 1}

Los actores involucrados presentaron unanimidad al manifestar su percepción frente a la clase tradicional; principalmente, solicitan rediseñar las metodologías típicas que no han evolucionado y recomiendan, en todas las técnicas abordadas, emplear tecnología como medio en el proceso de enseñanza y aprendizaje. De lo contrario, el nivel de motivación y compromiso (engagement) seguirá siendo muy bajo; esta situación afecta de forma directa a la población implicada.

Existe una relación directa entre el uso pedagógico de las Tic y la motivación de estudiantes en distintos procesos académicos. Esto se refleja no solo en el trabajo de campo del experimento, sino en el nivel de aprendizaje autónomo presentado en las posibilidades ofrecidas por el diseño pedagógico aplicado tanto en la institución como en otros escenarios virtuales. En síntesis, el uso pedagógico de las TIC influyó de forma significativa sobre la motivación de los estudiantes del grupo experimental y en las dinámicas propias de la clase; es claro que es un aspecto trascendental de desempeño de los estudiantes en las distintas áreas y escenarios de conocimiento.

\section{Objetivo específico 2}

La metodología empleada en el grupo experimental se enfatizó en la relación de contenidos con la realidad y el contexto de la población, tal como puede apreciarse, por ejemplo, en la observación participante holística. De ahí que el uso pedagógico de las TIC permitiera un alto nivel de resolución de dudas que se basaban de forma mayoritaria en la relación descrita.
El uso pedagógico de las Tic en la formación, ligado al proyecto "Empresarios en la Nube, Community Managers en la media técnica", permitió ahondar en la resolución de problemas cotidianos, y tener como eje medular las distintas circunstancias vinculadas a las empresas de los estudiantes. Con base en los resultados, se concluye que el diseño pedagógico aplicado propende a la identificación y la solución de problemas cotidianos vinculados con contenidos y prácticas propias de la clase. La resolución de problemas y el trabajo por proyectos -empresariales - aportó al aprendizaje significativo del grupo experimental, pues la prueba de salida así lo confirmó.

\section{Objetivo específico 3}

A diferencia de las conclusiones previas y no solo por los momentos de las sesiones de enfoque (focus group), sino por los resultados de encuestas, fue posible apreciar que en los hábitos de lectura y escritura no se notaron cambios significativos; por tanto, es claro que la estrategia de enseñanza y aprendizaje debía modificarse y tener en cuenta este importante componente. Desde la finalización de la investigación, hasta la publicación de este artículo, se han generado nuevos acercamientos de la población hacia la lectura y la escritura por medio de herramientas tecnológicas y metodologías innovadoras emergentes en el contexto internacional, como, por ejmplo, la gamificación y el uso pedagógico de redes sociales.

Ahora bien, en cuanto al pensamiento crítico, los cambios entre los momentos de entrada y salida para los dos grupos son significativos; el grupo experimental tuvo un alto grado de participación en todas las sesiones mediadas por el uso pedagógico de TIC; definitivamente, fueron muy importantes las ideas previas de estudiantes para establecer una sólida comparación al terminar cada una de las unidades de aprendizaje. Al personalizar el aprendizaje por medio de la metodología clase invertida (flipped learning) se abrieron nuevas posibilidades para los estudiantes para expresar opiniones y críticas sobre contenidos, casos y fenómenos inherentes a su realidad. 
Las preferencias a fin de acceder a la información variaron sustancialmente al emplear tecnología, tanto en el aula como fuera de ella; el alto nivel motivación por el aprendizaje autónomo generado por la metodología mencionada y la investigación en el aula permitieron que estos hábitos y comportamientos cambiaran de manera ostensible.

El uso de redes sociales, sitios web especializados y herramientas online para acceder a la información - desde diferentes dispositivos- en el grupo experimental también se transformó de forma significativa; se entendieron como grandes posibilidades para aprender y potenciar procesos académicos autónomos y ubicuos.

\section{Objetivo específico 4}

Esta conclusión se aborda desde dos puntos de vista. En primer lugar, la perspectiva unánime de estudiantes, padres y docentes en los dos momentos, al comprender el uso pedagógico de las TIC como medio ideal para mejorar el aprendizaje significativo de los estudiantes; claro está, con metodologías especializadas en ese uso. En segundo lugar, se hace referencia a los resultados de aprendizaje de la prueba de salida; la diferencia fue tan notoria que sorprendió al mismo investigador, ya que la forma como los estudiantes del grupo experimental apropiaron los contenidos a su realidad personal superó con creces al grupo de control. En esta investigación fue posible demostrar que el uso pedagógico de las TIC sí posibilitó un aprendizaje con significado para los estudiantes del grupo experimental de la muestra.

\section{Objetivo específico 5}

En la misma línea, pudo constatarse que las actividades tradicionales de tiempo libre se vieron afectadas por el método planteado. Un ejemplo es la disminución del número de horas de acceso a contenidos televisivos (los demás aspectos se describen en la discusión). A pesar de la gran posibilidad de que las redes sociales e internet pudieran conllevar a adicciones - como lo expresaron estudiantes, padres y docentes- el uso de tecnología para el aprendizaje en el tiempo libre se incrementó, así como el acceso a internet por medio de diferentes dispositivos.

Las actividades tradicionales fueron permeadas por nuevos usos de herramientas online y redes sociales; asimismo, tendencias de aprendizaje empleadas en clase como el uso de plataformas MOoc, tutoriales online y otros se convirtieron en prácticas cotidianas para los estudiantes del grupo experimental. Se concluye que el uso pedagógico de las TIC influyó de forma significativa en hábitos y actividades tradicionales desarrolladas en su tiempo libre.

Al revisar los resultados obtenidos, vinculados a estilos de aprendizaje, se aprecia la necesidad de los estudiantes por estar alejados de modelos pedagógicos que se basen en la pasividad y en la unidireccionalidad del flujo de información, convirtiéndolos en receptores; modelos que tienen en cuenta solo opiniones, contenidos y puntos de vista del docente y los consideran verdades. El modelo planteado tuvo influencia sobre las seis dimensiones de la variable dependiente y la medición de sus respectivos descriptores.

En fin, fue posible determinar, al culminar la investigación y con base en lo expuesto hasta este punto, que el uso pedagógico de las TIC influyó ostensiblemente en el aprendizaje significativo de los estudiantes de la media técnica de Zipaquirá, Colombia, al tener en cuenta las condiciones reales del contexto y aplicar una investigación de nivel cuasiexperimental con enfoque mixto.

\section{Referencias}

Ausubel, D. (1978). Psicología educativa. Un punto de vista cognoscitivo. México: Ed. Trillas.

Banoy, W. (2013). Canal de YouTube de Empresarios en la Nube, Community Managers en la Media Técnica [Archivos de video]. Recuperado de: https://www.youtube.com/channel/UCi8D2c6_Hd7tiL5wRVvb74g/videos?view_as=subscriber

Banoy, W. (2015). Empresarios en la Nube, community managers en la media técnica. Premio Compartir, mejores propuestas. Revista Palabra Maestra. Recuperado de: https://goo.gl/FwAmce

Bartolomé, A. \& Steffens, K. (2015). ¿Son los Mooc una alternativa de aprendizaje? Revista Científica de 
Educomunicación, 44(xxir), 91-99. Recuperado de: https://www.revistacomunicar.com/verpdf.php?numero $=44 \&$ articulo $=44-2015-10$

Cabero, J. \& Aguaded, J. (2013). Tecnologías y medios para la educación en la e-sociedad. Madrid: Alianza Editorial.

Cabiria, T. (2012). El "focus group": nuevo potencial de aplicación en el estudio de la acústica urbana. Athenea Digital. Revista de Pensamiento de Investigación Social, 2(XII), 129-152. Recuperado de: https://www.redalyc. org/pdf/537/53723279006.pdf

Cerda, H. (2008). Los elementos de la investigación; cómo reconocerlos, diseñarlos y construirlos ( $3^{\mathrm{a}} \mathrm{ed}$.). Bogotá: El Búho.

Corral, Y. (2009). Validez y confiabilidad de los instrumentos de investigación para la recolección de datos. Revista Ciencias de la Educación, 33(xIx), 228-247. Recuperado de: http://servicio.bc.uc.edu.ve/educacion/revista/ n33/art12.pdf

FNL (Flipped Learning Network). (2014). The four pillars of F-L-I-P. Recuperado de: https://flippedlearning.org/ wp-content/uploads/2016/07/PilaresFlip.pdf

Gardner. H. \& Davis, K. (2014). La generación APP, cómo los jóvenes gestionan su identidad, su privacidad y su imaginación en el mundo digital. Barcelona: Paidós.

Gómez, M., Deslauriers, J. \& Alzate, M. (2010). Cómo hacer tesis de maestría o doctorado, investigación, escritura y publicación. Bogotá: Ediciones ECOE.

Hernández, R., Fernández, C. \& Baptista, P. (2014). Metodología de la investigación (6 ${ }^{\mathrm{a}}$ ed.). México D. F: McGraw Hill Education.

Méndez, S. \& Cuevas, A. (2014). Manual introductorio al SPSS Statistics Standard Edition 22. Universidad de Celaya e Instituto Politécnico Nacional. Recuperado de: https://www.fibao.es/media/uploads/manual_de_ spss_universidad_de_celaya.pdf

Okuda, M. \& Gómez, C. (2005). Métodos en investigación cualitativa: triangulación. Revista Colombiana de Psi- quiatría, 1(xxxiv), 118-124. Recuperado de: http:// www.scielo.org.co/pdf/rcp/v34n1/v34n1a08.pdf

Otzen, T. \& Manterola, C. (2017). Técnicas de muestreo sobre una población a estudio. International Journal of Morphology, 35(1), 227-232. Recuperado de: https:// scielo.conicyt.cl/pdf/ijmorphol/v35n1/art37.pdf

Pereira, Z. (Enero-junio de 2011). Los diseños de método mixto en la investigación en educación: una experiencia concreta. Revista Electrónica Educare, xv(1), 15-29.

Pineda, E., de Alvarado, E. \& de Canales F. (1994). Metodología de la investigación. Wahington D. C.: Organización Panamericana de la Salud.

Ramírez, Y. y Rosas, D. (2014). Aplicación de la teoría de estilos de aprendizaje al diseño de contenidos didácticos en entornos virtuales. Revista Científica de Educación y Comunicación en la Sociedad del Conocimiento, 14(II), 176-197. Recuperado de: https://www. ugr.es/ sevimeco/revistaeticanet/numero142/Articulos/Formato/206.pdf

Robinson, K. (Febrero de 2006). Do schools kill creativity? [video online]. Recuperado de: https://www.ted.com/ talks/ken_robinson_says_schools_kill_creativity

Robinson, K. \& Aronica, L. (2015). Escuelas creativas, la revolución que está transformando la educación. Barcelona: Grijalbo.

Rojas, R. (2013). Guía para realizar investigaciones sociales. México, D. F: P y v Editores.

Sabariego, M. \& Bisquerra, R. (2004). El proceso de investigación (parte 1). En R. Bisquerra (coord), Metodología de la investigación educativa (pp. 89-125). Madrid: La Muralla.

Zárrate, A. (5 de mayo de 2016). Engagement educativo [Mensaje en blog]. Recuperado de: http://www.eleducador.com/engagement-educativo/

Zubiría, J. de. (2006). Los modelos pedagógicos, hacia una pedagogía dialogante. Bogotá: Editorial Magisterio; segunda edición. 